\title{
Preface for the Special Issue on Computational Techniques in Theoretical and Applied Statistics
}

\author{
Filipe J. Marques, Andriëtte Bekker, Mohammad Arashi
}

Recent technological developments and the exponential increase in computational power provide researchers in statistics with endless possibilities for developing methodologies and applying new techniques. This special issue, entitled "Computational Techniques in Theoretical and Applied Statistics", highlights some topics and specific problems, combining theoretical results, applications and computational techniques.

The works presented in this special issue cover different topics of research, as for example: Distribution Theory, Nonparametric Inference, Shrinkage/Penalized Estimation Strategies, Actuarial Sciences, Survival Analysis, and etc. In what follows we present a brief review of the various works published in this special issue.

In Survival Analysis, Balakrishnan and Feng introduced a Conway- Maxwell- Poisson Cure rate model under a proportional odds assumption for lifetime of susceptibles, with the baseline either the Weibull or the logistic distribution. A computationally efficient EM algorithm is presented, followed by an extensive Monte Carlo simulation study to illustrate the performance of this flexible model.

In the topic of Distribution Theory, Marques developed near-exact approximations for the linear combination of independent Logistic random variables which are based on the difference of two independent Generalized Integer Gamma distributions. The practical implementation of these distributions is only possible using software with high precision. In Bekker and Ferreira, the paper starts with a description of the setting. Bivariate gamma type distributions, within the elliptical framework are proposed, with the bivariate Nakagami distribution that is well known in the communications systems domain to describe the signal behaviour of fading channel, as a special case. Interesting observations follow from the calculated values of the outage probability and Laplace expressions. This work has a significant impact on the assumption of the underlying normality that is pervasive in the current literature in the communications systems field. In Nunes et al., theoretical results about limiting distributions of asymptotically linear statistics are demonstrated and applied through the use of computational simulations.

In $\mathrm{Wu}$ et al., the authors considered the problem in Actuarial Sciences of modelling aggregate claims with a dependence structure. The authors combine theoretical results for the aggregate claim distributions with computational algorithms for its implementation and present numerical studies for a specific case.

In the topic of Shrinkage/Penalized Estimation Strategies, Saleh and Norouzirad, proposed a closed form modified LASSO in multiple regression and compared its performance with shrinkage estimators. More precisely, using the principle of marginal distribution theory, shrinkage and penalized estimation strategies are developed for a non-orthogonal design matrix. Hence, a closed form modified LASSO estimator is proposed for the non-orthogonal design. In an extensive simulation study, the performance of shrinkage estimators is compared with the ridge regression and modified LASSO estimators. The computational aspect of this study is on finding the upper bound of risk functions and theoretical comparisons. In Emami and Kiani, shrinkage differenced based linear unified (Liu) estimators are proposed in semiparametric linear models, when multicollinearity is present. Exact characteristics of the estimators are derived and the region of optimality of each estimator is explored. These attempts formed the computational framework of this study, in the field of shrinkage estimation with multicollinearity problem. In Kazemi et al., a procedure is developed for ultra-high dimensional additive models, to identify nonzero and linear components. Hence a sure independence screening procedure based on the distance correlation between predictors and marginal distribution function of the response variable are proposed. Afterwards, penalized estimation techniques are used to identify nonzero and linear components, simultaneously. In this piece of work, complex computational aspects are revealed by proposing the procedures in ultra-high dimensions and algorithms used for

ISSN 2310-5070 (online) ISSN 2311-004X (print)

Copyright (C) 2018 International Academic Press 
finding tuning parameters. Still in the context of shrinkage/penalized estimation, Roozbeh, proposed a generalized ridge estimator in the high dimensional regression model. Asymptotic performance of this estimator, with growing dimensionality, is analyzed, and the performance is improved by shrinking it towards zero. Extensive computations is carried out to evaluate the efficiency of the proposed estimators in high dimension.

In the topic of Non-parametric Inference, Muhammad et al., developed non-parametric predictive inference for best linear combination of two biomarkers using parametric copulas. The computational advantages are presented in: i) the computations to find the optimal linear combination coefficient, ii) the implementation of the new methodology developed which combines non-parametric predictive inference for the marginals with an estimated parametric copula, and iii) finally, in the simulation studies developed.

Finally, using point estimation methods and re-sampling techniques, Visagie demonstrated with extensive computation that the parameter estimates obtained using the maximum likelihood and the empirical characteristic function estimators vary wildly in cases where the data are realized from a distribution resembling a limit case of the distribution containing fewer parameters. Notwithstanding the variability between estimates, in many cases the density functions achieved using various estimators, are almost identical in spite of substantially different parameter estimates.

Filipe J. Marques, Mathematics Department and Center for Mathematics and Its Applications, CMA/FCT, Faculdade de Ciências e Tecnologia, Universidade Nova de Lisboa, Portugal

Andriëtte Bekker, Department of Statistics, University of Pretoria, South Africa

Mohammad Arashi, Department of Statistics, Faculty of Mathematical Sciences, Shahrood University of Technology, Iran 